\title{
Nonlinear parameter and state estimation for cooperative systems in a bounded-error context
}

\author{
Michel Kieffer and Eric Walter \\ Laboratoire des Signaux et Systèmes \\ CNRS - Supélec - Université Paris-Sud \\ Plateau de Moulon, F-91192 Gif-sur-Yvette, France \\ \{kieffer, walter\}@lss.supelec.fr
}

\begin{abstract}
This paper is about guaranteed nonlinear parameter and state estimation. Sets are computed that contain all possible values of the parameter (or state) vector given bounds on the acceptable errors. The main requirement is that the dynamical equations describing the evolution of the model can be bounded between cooperatives models, i.e., models such that the off-diagonal entries of their Jacobian matrix remain positive. The performances and limitations of the techniques proposed are illustrated on a nonlinear compartmental model.
\end{abstract}

\section{Introduction}

Parameter and state estimation problems are encountered when modeling a process that involves uncertain quantities to be estimated from measurements.

Consider a system with known input vector $\mathbf{u}(t)$ and output vector $\mathbf{y}(t)$. Assume it is described by a model with the same input and consisting of a dynamical state equation

$$
\mathbf{x}^{\prime}(t)=\mathbf{f}(\mathbf{x}(t), \mathbf{p}, \mathbf{w}(t), \mathbf{u}(t)),
$$

with initial condition

$$
\mathbf{x}(0)=\mathbf{x}_{0}(\mathbf{p}),
$$

and an observation equation

$$
\mathbf{y}_{\mathrm{m}}(\mathbf{x}(t), \mathbf{p}, t)=\mathbf{g}(\mathbf{x}(t), \mathbf{p})+\mathbf{v}(t),
$$

where the vector $\mathbf{x}$ is the state of the model, $\mathbf{x}^{\prime}$ is its derivative with respect to time, $\mathbf{p}$ is a vector of unknown parameters and $\mathbf{w}$ and $\mathbf{v}$ are vectors of state perturbations and measurement noise. State perturbations account for the fact that (1) is only an approximation of reality. Measurement noise is introduced in (3) to represent the imperfection of the sensors measuring the outputs of the system.

Finding an estimate $\widehat{\mathbf{p}}$ for $\mathbf{p}$ such that the output of the model $\mathbf{y}_{\mathrm{m}}(\mathbf{x}(t), \mathbf{p}, t)$ is an acceptable approximation of the output of the system $\mathbf{y}(t)$ is called parameter estimation. Similarly, finding an estimate $\widehat{\mathbf{x}}(t)$ for $\mathbf{x}(t)$ is called state estimation. When the two problems are solved simultaneously, one speaks of joint parameter and state estimation. 
This paper focuses on bounded-error estimation. In this context, it is assumed that the pertubations and noise are bounded with known bounds and one looks for the set of all parameter (or state) vectors that are consistent with the experimental data and these bounds. Specific methods are available for the case where the model output is linear in the parameter vector (or the initial state vector), and we shall concentrate on the more difficult nonlinear case.

The first part of this paper deals with recursive state estimation of a continuoustime model assuming that the system output is measured at discrete time instants. An idealized algorithm is presented first. As the Kalman filter, it alternates prediction and correction. The prediction step computes the evolution of the set corresponding to the state estimate. The correction step takes place as soon as a measurement of the system output becomes available. It computes the intersection of the previously calculated set with the set of all state vectors that are consistent with this measurement and the bounds on the measurement error.

The second part of this paper deals with parameter estimation. It is much shorter since the same type of tools are used as in the correction step of state estimation.

The specific difficulty when estimating the parameter or state vector of a continuoustime state-space model is that most often there is no closed-form solution of the differential state equation, which complicates the required obtention of an inclusion function for this solution. Guaranteed interval integration could in principle be used, but it becomes notoriously pessimistic as soon as the uncertainty in the parameters and initial conditions is large, as required here. We shall see that a much less pessimistic numerical inclusion function for the model output can be evaluated if the differential model can be enclosed between two cooperative systems.

State and parameter estimations are illustrated with compartmental models, widely used in biology.

\section{Recursive state estimation}

\subsection{Introduction}

In this section, an estimate $\widehat{\mathbf{x}}(t)$ for $\mathbf{x}(t)$ is to be obtained such that $\mathbf{y}_{\mathrm{m}}(\mathbf{x}(t), \mathbf{p}, t)$, the output of the model $(1)-(3)$, is an acceptable approximation of the output $\mathbf{y}(t)$ of the system.

Note that the parameter vector $\mathbf{p}$ is not necessarily known. Two approaches may be considered to estimating $\mathbf{x}(t)$ when $\mathbf{p}$ is uncertain. A first method assumes some prior knowledge about the evolution of $\mathbf{p}$, described by the differential equation

$$
\mathbf{p}^{\prime}(t)=\mathbf{f}_{\mathrm{p}}\left(\mathbf{x}(t), \mathbf{p}(t), \mathbf{w}_{\mathrm{p}}(t), \mathbf{u}(t)\right), \mathbf{p}(0)=\mathbf{p}_{0},
$$

where $\mathbf{w}_{\mathrm{p}}$ plays the same role as $\mathbf{w}$ for $\mathbf{x}$. (If the parameters are assumed to be constant, the differential equation in (4) boils down to $\mathbf{p}^{\prime}(t)=\mathbf{0}$.) Defining an extended state vector

$$
\mathbf{x}^{\mathrm{e}}(t)=\left(\mathbf{x}^{\mathrm{T}}(t), \mathbf{p}^{\mathrm{T}}(t)\right)^{\mathrm{T}}
$$


makes it possible to obtain from (1) and (4) an extended dynamical state equation

$$
\left(\begin{array}{l}
\mathbf{x}(t) \\
\mathbf{p}(t)
\end{array}\right)^{\prime}=\left(\begin{array}{l}
\mathbf{f}(\mathbf{x}(t), \mathbf{p}, \mathbf{w}(t), \mathbf{u}(t)) \\
\mathbf{f}_{\mathbf{p}}\left(\mathbf{x}(t), \mathbf{p}(t), \mathbf{w}_{\mathrm{p}}(t), \mathbf{u}(t)\right)
\end{array}\right),\left(\begin{array}{c}
\mathbf{x}(0) \\
\mathbf{p}(0)
\end{array}\right)=\left(\begin{array}{c}
\mathbf{x}_{0}\left(\mathbf{p}_{0}\right) \\
\mathbf{p}_{0}
\end{array}\right)
$$

or equivalently

$$
\left(\mathbf{x}^{\mathrm{e}}(t)\right)^{\prime}=\mathbf{f}^{\mathrm{e}}\left(\mathbf{x}^{\mathrm{e}}(t), \mathbf{w}(t), \mathbf{w}_{\mathrm{p}}(t), \mathbf{u}(t)\right), \mathbf{x}^{\mathrm{e}}(0)=\mathbf{x}_{0}^{\mathrm{e}}\left(\mathbf{p}_{0}\right) .
$$

With this approach, which corresponds to joint parameter and state estimation, the distinction between state variables and parameters disappears, and the situation is formally equivalent to the case with no uncertain parameters.

A second method, which is the one to be employed in this paper, integrates the uncertainty about $\mathbf{p}$ in the state perturbations and measurement noise. No attempt will then be made at estimating $\mathbf{p}$, which will be considered as a nuisance parameter vector.

When $\mathbf{f}$ and $\mathbf{g}$ in (1) and (3) are linear functions of the state vector and when moreover the perturbations and noise are additive and receive a probabilistic description by their means and covariances, Kalman filtering [15] is the standard approach to state estimation. In the context of bounded errors, many tools are also available, see, e.g., [2], [20] and [23].

In a nonlinear context, the methodology is far less developed. When uncertainty is explicitly taken into account, this is most often by using an extended Kalman filter [4] based on the linearization of (1) around the state trajectory. It is well known that this type of filter may fail to produce a useful estimate of the state vector, and that the characterization of the uncertainty in this estimate is not reliable.

Guaranteed state bounding is an attractive alternative, which has been considered in a discrete-time context in [11] and [18]. All state vectors consistent with the data, model and bounds are enclosed in a subpaving, consisting of a union of disconnected boxes. In a continuous-time context, a state estimator for models such as that described by (1) and (3) was proposed in [10] but with no state perturbation or parameter uncertainty taken into account. Techniques bounding the state of continuous-time systems with poorly known state equations and inputs are presented in [1] and [6], with applications in waste processing. Provided specific assumptions are satisfied by the signs of the entries of $\partial \mathbf{f} / \partial \mathbf{x}$, interval observers can be built. An interval observer is a pair of classical point observers computing a box enclosure of the state $\mathbf{x}$ at any given time based on lower and upper bounds for each of the uncertain variables.

In this paper, interval observers and the recursive state estimation algorithm presented in [12] and [18] are combined to enclose the state $\mathbf{x}(t)$ of the model (1) - (3) at any given instant of time $t$ in a subpaving. This is performed recursively and can thus be implemented in real time. Preliminary results have been presented in [19].

An idealized algorithm is first proposed in Section 2.2. An implementable counterpart of this algorithm is then described in Section 2.3. The advantages and limitations of the approach are illustrated on an example in Section 2.4.

\subsection{Idealized algorithm}

Consider the model $(1)-(3)$ and a set of sampling instants $\mathscr{T}=\left\{t_{i}\right\}_{i \in \mathbb{N}^{*}}$, such that $t_{i+1}>t_{i}$, at which the measurements $\mathbf{y}\left(t_{i}\right)$ have been collected. Initially, $\mathbf{x}(0)$ is only 
known to belong to some box $\left[\mathbf{x}_{0}\right]$. The vector $\mathbf{p}$ of uncertain parameters is assumed to be constant and to belong to some known prior box $\left[\mathbf{p}_{0}\right]$. The state perturbation $\mathbf{w}(t)$ is assumed to satisfy $\underline{\mathbf{w}}(t) \leqslant \mathbf{w}(t) \leqslant \overline{\mathbf{w}}(t)$ at any $t \geqslant 0$, where $[\mathbf{w}(t)]=[\underline{\mathbf{w}}(t), \overline{\mathbf{w}}(t)]$ is known for all $t$ and the inequalities are to be understood componentwise. The measurement noise $\mathbf{v}\left(t_{i}\right)$ is similarly assumed to belong to $\left[\mathbf{v}\left(t_{i}\right)\right]=\left[\underline{\mathbf{v}}\left(t_{i}\right), \overline{\mathbf{v}}\left(t_{i}\right)\right]$, known at each $t_{i}$. The information $\mathscr{I}(t)$ available at time $t \geqslant 0$ is given by

$$
\mathscr{I}(t)=\left\{\left[\mathbf{x}_{0}\right],\left[\mathbf{p}_{0}\right],\{[\mathbf{w}(\tau)], \mathbf{u}(\tau)\}_{\tau \in[0, t]},\left\{\left[\mathbf{v}\left(t_{i}\right)\right]\right\}_{i=1}^{M}\right\}
$$

where $t_{M}$ is such that $t_{M} \leqslant t<t_{M+1}$. In this context, causal state estimation is the caracterization of the set $\mathscr{X}(t)$ of all values of the state $\mathbf{x}(t)$ at any time $t \geqslant 0$ that are consistent with $\mathscr{I}(t)$.

As in the Kalman filter, the idealized recursive causal state estimator consists of two steps.

For the prediction step, assume that $\mathscr{X}\left(t_{i}\right) \subset \mathscr{D}$ is some set guaranteed to contain $\mathbf{x}\left(t_{i}\right)$. For any given $\mathbf{x} \in \mathscr{X}\left(t_{i}\right)$, let $\varphi\left(\mathbf{x}, t, t_{i}, \mathbf{p},\{\mathbf{w}(\tau), \mathbf{u}(\tau)\}_{\tau \in\left[t_{i}, t\right]}\right)$ be the value at time $t$ of the flow associated with (1) that coincides with $\mathbf{x}$ at time $t_{i}$. Define the predicted set $\mathscr{X}^{+}\left(t_{i+1}\right)$ as

$$
\begin{aligned}
\mathscr{X}^{+}\left(t_{i+1}\right)=\{ & \varphi\left(\mathbf{x}, t_{i+1}, t_{i}, \mathbf{p},\{\mathbf{w}(\tau), \mathbf{u}(\tau)\}_{\tau \in\left[t_{i}, t_{i+1}\right]}\right) \\
& \left.\mid \mathbf{p} \in\left[\mathbf{p}_{0}\right], \mathbf{w}(\tau) \in[\mathbf{w}(\tau)], \mathbf{x} \in \mathscr{X}\left(t_{i}\right), \tau \in\left[t_{i}, t_{i+1}\right]\right\} .
\end{aligned}
$$

By construction, $\mathbf{x}\left(t_{i+1}\right) \in \mathscr{X}^{+}\left(t_{i+1}\right)$.

Now, for the correction step, let $\left[\mathbf{y}\left(t_{i+1}\right)\right]$ be the box containing all possible values of the noise-free output when the value of the measured output is $\mathbf{y}\left(t_{i+1}\right)$

$$
\left[\mathbf{y}\left(t_{i+1}\right)\right]=\mathbf{y}\left(t_{i+1}\right)-\left[\mathbf{v}\left(t_{i+1}\right)\right],
$$

and let $\mathscr{X}^{\mathrm{o}}\left(t_{i+1}\right)$ be the set of all values of the state at time $t_{i+1}$ that could have led to an observation $\mathbf{y}$ in $\left[\mathbf{y}\left(t_{i+1}\right)\right]$

$$
\mathscr{X}^{\mathrm{o}}\left(t_{i+1}\right)=\left\{\mathbf{x} \in \mathbb{R}^{n} \mid \mathbf{g}(\mathbf{x}, \mathbf{p}) \in\left[\mathbf{y}\left(t_{i+1}\right)\right], \mathbf{p} \in\left[\mathbf{p}_{0}\right]\right\} .
$$

Then, the corrected set

$$
\mathscr{X}\left(t_{i+1}\right)=\mathscr{X}^{+}\left(t_{i+1}\right) \cap \mathscr{X}^{\mathrm{o}}\left(t_{i+1}\right)
$$

is also guaranteed to contain $\mathbf{x}\left(t_{i+1}\right)$ (see Figure 1).

This is summarized in the following idealized algorithm.

\section{Algorithm 1}

For $i=0$ to $N-1$, do \{

1. Prediction: evaluate $\mathscr{X}^{+}\left(t_{i+1}\right)$;

2. Correction: $\left.\mathscr{X}\left(t_{i+1}\right)=\mathscr{X}^{+}\left(t_{i+1}\right) \cap \mathscr{X}^{o}\left(t_{i+1}\right) ;\right\}$

It is easy to show [16] that $\mathscr{X}(t)$ as evaluated by Algorithm 1 is the smallest set guaranteed to contain $\mathbf{x}(t)$ that can be computed from $\mathscr{I}(t)$ and (1). The next section presents the basic tools required to obtain an implementable counterpart to Algorithm 1. 


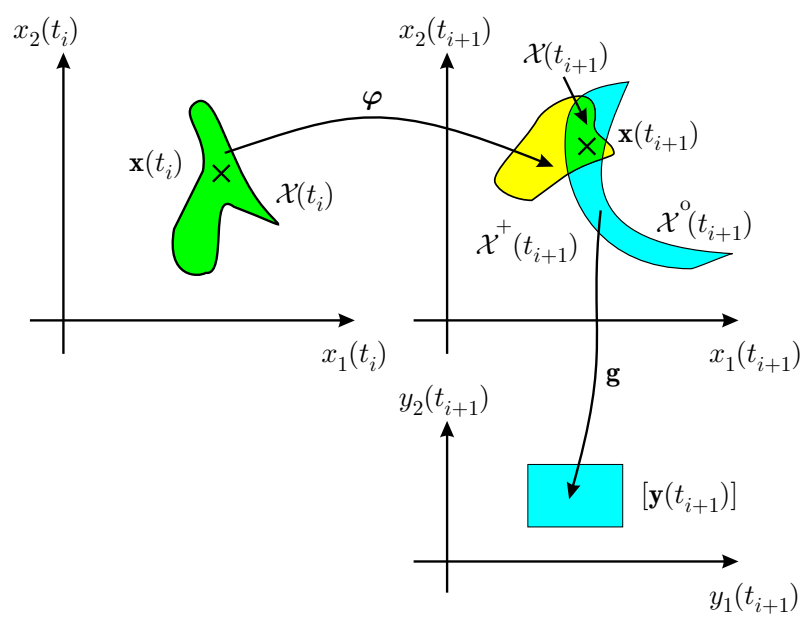

Fig. 1. Idealized state estimation

\subsection{Implementation issues}

To obtain an implementable counterpart to Algorithm 1, three main problems have to be solved.

The first one is to represent the sets $\mathscr{X}\left(t_{i}\right), \mathscr{X}^{\mathrm{o}}\left(t_{i}\right)$ and $\mathscr{X}^{+}\left(t_{i}\right)$ in computer memory. In this paper, the description of sets using subpavings presented in [17] is used.

The second problem is the evaluation of $\mathscr{X}^{\mathrm{o}}\left(t_{i+1}\right)$ during the correction step (8). An outer approximation $\widehat{\mathscr{X}^{\mathrm{o}}}\left(t_{i+1}\right)$ of $\mathscr{X}^{\mathrm{o}}\left(t_{i+1}\right)$ by a subpaving can be obtained using the SIVIA algorithm (see below). The precision of this outer approximation is controlled by a precision factor $\varepsilon_{S}$.

The remaining problem is the solution at the prediction step of the set of IVPs required to evaluate $\mathscr{X}^{+}\left(t_{i+1}\right)$. Standard guaranteed tools are available to solve IVPs such as $\left\{\mathbf{x}^{\prime}=\mathbf{f}(\mathbf{x}, t), \mathbf{x}(0)=\mathbf{x}\right\}$ or $\left\{\mathbf{x}^{\prime}=\mathbf{f}(\mathbf{x}, t), \mathbf{x}(0) \in[\mathbf{x}]\right\}$, see, e.g., AWA ([21], [22]), COSY ([8], [9]) or VNODE ([24]). These techniques are based on Brouwer's fixedpoint theorem and build a Taylor expansion of the solution while bounding the remainder in a guaranteed way. However, they become very inefficient in the presence of unknown parameters or bounded state perturbations because the bounds on the remainder soon become extremely large. We shall present a more efficient approach, based on cooperativity.

SIVIA Using interval analysis, it is possible to provide inner and outer approximations of the set $\mathscr{X}^{\circ}\left(t_{i+1}\right)$ defined by (8), using the algorithm SIVIA (for Set Inverter Via Interval Analysis, see [13] and [14]) briefly recalled here.

An initial bounded search set $\mathbb{X}^{\mathrm{o}}$ guaranteed to contain $\mathscr{X}^{\mathrm{o}}\left(t_{i+1}\right)$ has to be provided first. SIVIA partitions $\mathbb{X}^{0}$ into three subpavings, namely $\mathbb{X}_{\text {in }}$ contained in $\mathscr{X}^{\circ}\left(t_{i+1}\right)$, $\mathbb{X}_{\text {out }}$ such that its intersection with $\mathscr{X}^{\circ}\left(t_{i+1}\right)$ is empty and $\mathbb{X}_{\text {bound }}$ for which no conclusion could be reached. 
Consider a box $[\mathbf{x}] \subset \mathbb{X}^{0}$ and let $[\mathbf{g}]($.$) be an inclusion function for \mathbf{g}($.$) .$

1. If $[\mathbf{g}]\left([\mathbf{x}],\left[\mathbf{p}_{0}\right]\right) \subset\left[\mathbf{y}\left(t_{i+1}\right)\right]$, then for any $\mathbf{x} \in[\mathbf{x}]$ and $\mathbf{p} \in\left[\mathbf{p}_{0}\right], \mathbf{g}(\mathbf{x}, \mathbf{p}) \in\left[\mathbf{y}\left(t_{i+1}\right)\right]$ and $[\mathbf{x}]$ is entirely included in $\mathscr{X}^{\mathrm{o}}\left(t_{i+1}\right)$; it is thus stored in $\mathbb{X}_{\mathrm{in}}$.

2. If $[\mathbf{g}]\left([\mathbf{x}],\left[\mathbf{p}_{0}\right]\right) \cap\left[\mathbf{y}\left(t_{i+1}\right)\right]=\emptyset$, then $\mathbf{g}\left([\mathbf{x}],\left[\mathbf{p}_{0}\right]\right) \cap\left[\mathbf{y}\left(t_{i+1}\right)\right]=\emptyset$ and $[\mathbf{x}]$, proved to have an empty intersection with $\mathscr{X}^{\mathrm{o}}\left(t_{i+1}\right)$, can be stored in $\mathbb{X}_{\text {out }}$.

3. If neither of the previous tests is satisfied, then $[\mathbf{x}]$ is undetermined. If the width of such an undetermined box is larger than the precision factor $\varepsilon_{\mathrm{S}}$, then it is bisected into two subboxes $\left[\mathbf{x}_{1}\right]$ and $\left[\mathbf{x}_{2}\right]$ to which the same tests are applied. Undetermined boxes that are too small to be bisected are stored into $\mathbb{X}_{\text {bound }}$.

$\mathscr{X}^{\mathrm{o}}\left(t_{i+1}\right)$ is thus bracketed (in the sense of inclusion) between $\mathbb{X}_{\text {in }}$ and $\widehat{\mathscr{X}}^{\mathrm{o}}\left(t_{i+1}\right)=$ $\mathbb{X}_{\text {in }} \cup \mathbb{X}_{\text {bound }}$. The volume of the uncertainty subpaving $\mathbb{X}_{\text {bound }}$ may be reduced, at the cost of increasing computational effort. Note that there is actually no need to store $\mathbb{X}_{\text {out }}$.

Inclusion functions based on cooperativity This section aims at defining an implementable procedure for computing $\mathscr{X}^{+}\left(t_{i+1}\right)$ defined by $(6)$ based on the concept of cooperativity [26].

Definition 1. A dynamical system

$$
\mathbf{x}^{\prime}=\mathbf{f}(\mathbf{x}, t)
$$

is cooperative over a domain $\mathscr{D}$ if

$$
\frac{\partial f_{i}}{\partial x_{j}}(\mathbf{x}, t) \geqslant 0, \text { for all } i \neq j, t \geqslant 0 \text { and } \mathbf{x} \in \mathscr{D},
$$

i.e., if all off-diagonal entries of the Jacobian matrix of $\mathbf{f}$ are non-negative for all $t \geqslant 0$ and $\mathbf{x} \in \mathscr{D}$.

The following theorem, which is a reformulation of a result in [26], will be used to obtain an enclosure for $\mathbf{x}(t)$ in (1). This enclosure will be instrumental in the implementation of the prediction step.

Theorem 1. If there exists a pair of cooperative systems

$$
\underline{\mathbf{x}}^{\prime}=\underline{\mathbf{f}}(\underline{\mathbf{x}}, \underline{\mathbf{p}}, \overline{\mathbf{p}}, t) \text { and } \overline{\mathbf{x}}^{\prime}=\overline{\mathbf{f}}(\overline{\mathbf{x}}, \underline{\mathbf{p}}, \overline{\mathbf{p}}, t)
$$

satisfying

$$
\underline{\mathbf{x}}_{0} \leqslant \mathbf{x}(0) \leqslant \overline{\mathbf{x}}_{0}
$$

and

$$
\underline{\mathbf{f}}(\underline{\mathbf{x}}, \underline{\mathbf{p}}, \overline{\mathbf{p}}, t) \leqslant \mathbf{f}(\mathbf{x}, \mathbf{p}, \mathbf{w}, \mathbf{u}) \leqslant \overline{\mathbf{f}}(\overline{\mathbf{x}}, \underline{\mathbf{p}}, \overline{\mathbf{p}}, t),
$$

for all $\mathbf{p} \in[\underline{\mathbf{p}}, \overline{\mathbf{p}}], \mathbf{w}(t) \in[\underline{\mathbf{w}}(t), \overline{\mathbf{w}}(t)], t \geqslant 0$ and $\mathbf{x} \in \mathscr{D}$ then the state of (1) satisfies

$$
\underline{\mathbf{x}}(t) \leqslant \mathbf{x}(t) \leqslant \overline{\mathbf{x}}(t), \text { for all } t \geqslant 0,
$$

where $\underline{\mathbf{x}}(t)=\underline{\phi}\left(\underline{\mathbf{x}}_{0}, \underline{\mathbf{p}}, \overline{\mathbf{p}}, t\right)$ is the flow associated with $\left\{\underline{\mathbf{x}}^{\prime}=\underline{\mathbf{f}}(\underline{\mathbf{x}}, \underline{\mathbf{p}}, \overline{\mathbf{p}}, t), \underline{\mathbf{x}}(0)=\underline{\mathbf{x}}_{0}\right\}$ and $\overline{\mathbf{x}}(t)=\bar{\phi}\left(\overline{\mathbf{x}}_{0}, \underline{\mathbf{p}}, \overline{\mathbf{p}}, t\right)$ is the flow associated with $\left\{\overline{\mathbf{x}}^{\prime}=\overline{\mathbf{f}}(\overline{\mathbf{x}}, \underline{\mathbf{p}}, \overline{\mathbf{p}}, t), \overline{\mathbf{x}}(0)=\overline{\mathbf{x}}_{0}\right\}$. 
For any $t \geqslant 0$, the box-valued function

$$
[\phi]\left(\underline{\mathbf{x}}_{0}, \overline{\mathbf{x}}_{0}, \underline{\mathbf{p}}, \overline{\mathbf{p}}, t\right)=\left[\underline{\phi}\left(\underline{\mathbf{x}}_{0}, \underline{\mathbf{p}}, \overline{\mathbf{p}}, t\right), \bar{\phi}\left(\overline{\mathbf{x}}_{0}, \underline{\mathbf{p}}, \overline{\mathbf{p}}, t\right)\right]
$$

is thus an inclusion function for $\mathbf{x}(t)$, the solution of (1). However, this function is difficult to evaluate, as usually no explicit expressions are available for $\phi($.$) and \bar{\phi}($.$) .$ Interval analysis provides tools for computing guaranteed outer approximations of the solution of initial value problems, see, e.g., [24]. Using these techniques, it becomes possible to compute tight enclosures of $\underline{\phi}\left(\underline{\mathbf{x}}_{0}, \underline{\mathbf{p}}, \overline{\mathbf{p}}, t\right)$ and $\bar{\phi}\left(\overline{\mathbf{x}}_{0}, \underline{\mathbf{p}}, \overline{\mathbf{p}}, t\right)$ as

$$
\left[\underline{\phi}\left(\underline{\mathbf{x}}_{0}, \underline{\mathbf{p}}, \overline{\mathbf{p}}, t\right)\right]=\left[\underline{\underline{\phi}}\left(\underline{\mathbf{x}}_{0}, \underline{\mathbf{p}}, \overline{\mathbf{p}}, t\right), \overline{\phi\left(\underline{\mathbf{x}}_{0}, \underline{\mathbf{p}}, \overline{\mathbf{p}}, t\right)}\right]
$$

and

The function

$$
\left[\bar{\phi}\left(\overline{\mathbf{x}}_{0}, \underline{\mathbf{p}}, \overline{\mathbf{p}}, t\right)\right]=\left[\bar{\phi}\left(\overline{\mathbf{x}}_{0}, \underline{\mathbf{p}}, \overline{\mathbf{p}}, t\right), \overline{\bar{\phi}\left(\overline{\mathbf{x}}_{0}, \underline{\mathbf{p}}, \overline{\mathbf{p}}, t\right)}\right] .
$$

$$
[[\phi]]\left(\left[\mathbf{x}_{0}\right],\left[\mathbf{p}_{0}\right], t\right)=\left[\underline{\underline{\phi}}\left(\underline{\mathbf{x}_{0}}, \underline{\mathbf{p}}, \overline{\mathbf{p}}, t\right), \overline{\bar{\phi}\left(\overline{\mathbf{x}}_{0}, \underline{\mathbf{p}}, \overline{\mathbf{p}}, t\right)}\right]
$$

is thus such that

$$
\varphi\left(\mathbf{x}, t, 0, \mathbf{p},\{\mathbf{w}(\tau), \mathbf{u}(\tau)\}_{\tau \in[0, t]}\right) \in[[\phi]]\left(\left[\mathbf{x}_{0}\right],\left[\mathbf{p}_{0}\right], t\right),
$$

for $\mathbf{x}_{0} \in\left[\mathbf{x}_{0}\right], \mathbf{p} \in[\underline{\mathbf{p}}, \overline{\mathbf{p}}], \mathbf{w}(t) \in[\underline{\mathbf{w}}(t), \overline{\mathbf{w}}(t)], t \geqslant 0$, and is therefore an inclusion function for the solution $\mathbf{x}(t)$ of $(1)$, which can be numerically evaluated for any $t \geqslant 0$.

Let $\Phi\left(\left[\mathbf{x}_{0}\right],\left[\mathbf{p}_{0}\right], t\right)$ be the set of all $\mathbf{x}(t)$ that can be traced back to an initial condition in $\left[\mathbf{x}_{0}\right]$ according to (1) with a parameter vector $\mathbf{p} \in\left[\mathbf{p}_{0}\right]$. Then if the conditions of Theorem 1 are verified

$$
\mathbf{x}(t) \in \Phi\left(\left[\mathbf{x}_{0}\right],\left[\mathbf{p}_{0}\right], t\right) \subset[[\phi]]\left(\left[\mathbf{x}_{0}\right],\left[\mathbf{p}_{0}\right], t\right) \text { for any } t \geqslant 0 .
$$

Interval observers using $[[\phi]]\left(\left[\mathbf{x}_{0}\right],\left[\mathbf{p}_{0}\right], t\right)$ are only able to provide a box containing $\Phi\left(\left[\mathbf{x}_{0}\right],\left[\mathbf{p}_{0}\right], t\right)$. However, $\Phi\left(\left[\mathbf{x}_{0}\right],\left[\mathbf{p}_{0}\right], t\right)$ is usually not a box, see Figure 2 . Here, we propose to improve the accuracy of the approximation of $\Phi\left(\left[\mathbf{x}_{0}\right],\left[\mathbf{p}_{0}\right], t\right)$ by enclosing it in a subpaving using the IMAGESP algorithm presented in [17] and [18] and briefly recalled now.

The algorithm IMAGESP consists of three steps. First, $\left[\mathbf{x}_{0}\right]$ is minced, i.e., divided into boxes of width less than a given precision factor $\varepsilon_{\mathrm{I}}$. Then, the images of all these boxes are evaluated using an inclusion function of $\Phi$ and stored into a list $\mathscr{L}$ of image boxes. Finally, all boxes in $\mathscr{L}$ are merged to obtain a subpaving guaranteed to contain $\Phi\left(\left[\mathbf{x}_{0}\right],\left[\mathbf{p}_{0}\right], t\right)$. The time needed to obtain this subpaving and the precision of the description (measured, e.g., using a Hausdorff distance to the approximated set) increase when the precision factor $\varepsilon_{\mathrm{I}}$ decreases.

The only requirement for IMAGESP is the availability of an inclusion function for $\Phi$, which is obtained using $[[\phi]]$.

Remark 1. In the previous presentation, only $\left[\mathbf{x}_{0}\right]$ has been minced, but if one considered the extended state vector $\mathbf{x}^{\mathrm{e}}(t)=\left(\mathbf{x}^{\mathrm{T}}(t), \mathbf{p}^{\mathrm{T}}\right)^{\mathrm{T}}$, with initial condition $\mathbf{x}_{0}^{\mathrm{e}} \in\left[\mathbf{x}_{0}^{\mathrm{e}}\right]=$ $\left(\left[\mathbf{x}_{0}\right]^{\mathrm{T}},\left[\mathbf{p}_{0}\right]^{\mathrm{T}}\right)^{\mathrm{T}}$, the mincing could have been performed on $\left[\mathbf{x}_{0}^{\mathrm{e}}\right]$. When $\left[\mathbf{p}_{0}\right]$ is a nondegenerate interval, the resulting enclosure is usually more precise, but obtained with an increased computational effort. 


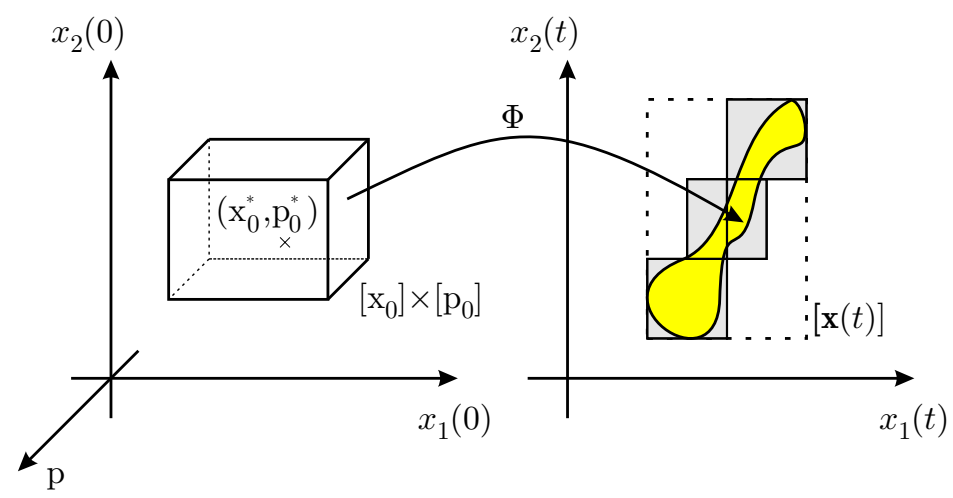

Fig. 2. State estimates obtained with an interval observer (box in dashed lines) and an approximate set observer (union of light grey boxes on the right)

Implementable algorithm Assume that $\mathscr{X}(t)$ has to be evaluated, with $t$ such that $t=t_{N}$ and that $\mathscr{X}(0)=\left[\mathbf{x}_{0}\right]$. The following algorithm is a counterpart to Algorithm 1.

\section{Algorithm 2}

For $i=0$ to $N-1$, do \{

1. Prediction: evaluate $\widehat{\mathscr{X}}^{+}\left(t_{i+1}\right)$ using IMAGESP;

2. Correction: evaluate $\widehat{\mathscr{X}}\left(t_{i+1}\right)$ using SIVIA with initial search domain $\widehat{\mathscr{X}}^{+}\left(t_{i+1}\right)$; \}

Convergence properties have been established in [14] for SIVIA and in [18] for IMAGESP. The convergence of Algorithm 2 depends not only on $\varepsilon_{\mathrm{S}}$ and $\varepsilon_{\mathrm{I}}$, but also on the quality of the enclosure of (1) provided by the pair of cooperative systems.

\subsection{Example}

Compartment models are frequently used in pharmacokinetics, chemistry or biology. They consist of a collection of tanks containing material. These tanks, represented by circles echange material between them and with the rest of the world, as materialized by arrows. Each tank is supposed to be homogeneous and the quantity of material in compartment $i$ is denoted by $x_{i}$. Many types of compartment models are available (see, e.g., [5]), but all share the property that the evolution of the quantities of material in the compartments is governed by a state equation that may easily be enclosed between cooperative models.

Assume that the evolution of the vector of quantities of material $\mathbf{x}=\left(x_{1}, x_{2}\right)^{\mathrm{T}}$ in the compartments of the model of Figure 3 is given by

$$
\left\{\begin{array}{l}
x_{1}^{\prime}=-\frac{p_{1} x_{1}}{1+p_{2} x_{1}}-p_{3} x_{1}+p_{4} x_{2}+u, \\
x_{2}^{\prime}=\frac{p_{1} x_{1}}{1+p_{2} x_{1}}-p_{4} x_{2},
\end{array}\right.
$$




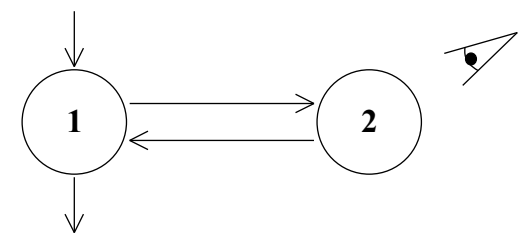

Fig. 3. Two-compartment model

and that only $x_{2}$ is measured, according to the measurement equation

$$
y\left(t_{i}\right)=\left(1+\varepsilon_{1}\right) x_{2}\left(t_{i}\right)
$$

where $\varepsilon_{1}$ is bounded.

All parameters are supposed to be known except for $p_{1} \in\left[p_{1}\right]=[0.9,1.1]$ and the initial state of the system is only known to belong to $\left[\mathbf{x}_{0}\right]=[0,1] \times[0,1]$. Data have been simulated with the actual value of the parameter vector $\mathbf{p}^{*}=(1,4 / 3,1 / 2,1 / 4)^{\mathrm{T}}$, $\mathbf{x}_{0}^{*}=(0,0)^{\mathrm{T}}$ and

$$
\left\{\begin{array}{l}
u(t)=1 \text { when } 0 \leqslant t<1 \text { and } 2.5 \leqslant t<3.5 \\
u(t)=0 \text { elsewhere. }
\end{array}\right.
$$

At 20 regularly-spaced time instants from 0.5 s to 10 s, a measurement of $x_{2}$ is taken and corrupted by a bounded relative noise $e_{1} \in[-0.1,0.1]$. The problem is to determine the set of all values of the state vector that are consistent with the model, the measurements and their uncertainty.

The dynamical model (12) can be bounded by the two models

$$
\left\{\begin{array}{l}
x_{1}^{\prime}=-\frac{\bar{p}_{1} x_{1}}{1+p_{2}^{*} x_{1}}-p_{3}^{*} x_{1}+p_{4}^{*} x_{2}+u \\
x_{2}^{\prime}=\frac{\underline{p}_{1} x_{1}}{1+p_{2}^{*} x_{1}}-p_{4}^{*} x_{2}
\end{array}\right.
$$

and

$$
\left\{\begin{array}{l}
x_{1}^{\prime}=-\frac{\underline{p}_{1} x_{1}}{1+p_{2}^{*} x_{1}}-p_{3}^{*} x_{1}+p_{4}^{*} x_{2}+u \\
x_{2}^{\prime}=\frac{\bar{p}_{1} x_{1}}{1+p_{2}^{*} x_{1}}-p_{4}^{*} x_{2}
\end{array}\right.
$$

which are easily proved to be cooperative, as the vector of quantities of material is positive. Moreover, as $\left[\mathbf{x}_{0}\right]=[0,1] \times[0,1]$, the conditions of Theorem 1 are satisfied. Thus, the prediction part of the recursive state estimation algorithm presented in Section 2.3 can be implemented using an inclusion function built from (14) and (15) and evaluated by guaranteed numerical integration.

The correction step involves the SIVIA algorithm presented in Section 2.3. The bounds for $v\left(t_{i}\right)$ in (3) are computed knowing that

$$
y\left(t_{i}\right)=\mathbf{g}\left(\mathbf{x}^{*}\left(t_{i}\right), \mathbf{p}^{*}\right)\left(1+e_{1}\left(t_{i}\right)\right)
$$


where $e_{1}\left(t_{i}\right)$ is a realization of a random variable with support restricted to $[-0.1,0.1]$ and

$$
\mathbf{g}(\mathbf{x}, \mathbf{p})=x_{2}
$$

Thus

$$
y\left(t_{i}\right) \in \mathbf{g}\left(\mathbf{x}^{*}\left(t_{i}\right), \mathbf{p}^{*}\right)(1+[-0.1,0.1])
$$

and

$$
\begin{aligned}
\mathbf{g}\left(\mathbf{x}^{*}\left(t_{i}\right), \mathbf{p}^{*}\right) & \in \frac{1}{1+[-0.1,0.1]} y\left(t_{i}\right) \\
& \in(1+[-0.081,0.112]) y\left(t_{i}\right)
\end{aligned}
$$

At each measurement time, the measurement noise is thus known to belong to

$$
\left[v\left(t_{i}\right)\right]=[-0.081,0.112] y\left(t_{i}\right) .
$$

All resulting intervals guaranteed to contain the noise-free output of the system are represented on Figure 4.

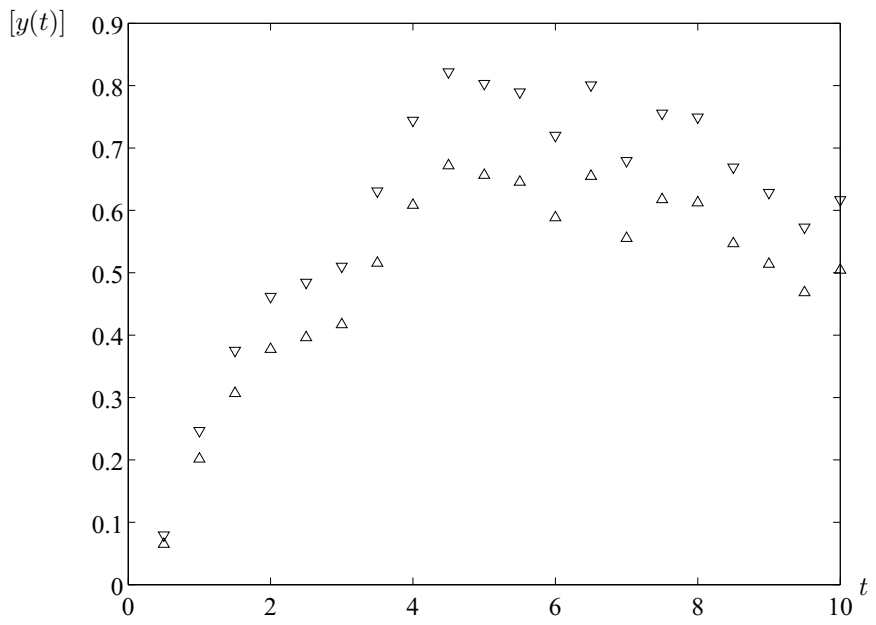

Fig. 4. Intervals guaranteed to contain the true values of $x_{2}\left(t_{i}\right)$

Two simulations have been performed, both with the algorithm described in Section 2.3, but with differing prediction step. The first one (Case $a$ ) is performed with a direct guaranteed integration of (12) taking into account the uncertain parameters. The second (Case $b$ ) involves an inclusion function built with (14) and (15). Guaranteed integration has been performed using the VNODE package, see [24]. The lower and upper bounds of the smallest boxes enclosing the predicted and corrected sets are represented for each measurement time on Figure 5 (for $x_{1}$, the lower bounds of the predicted 


\begin{tabular}{c|c|c|c} 
& Case $a$ & \multicolumn{2}{c}{ Case $b$} \\
\hline$\varepsilon_{\mathrm{S}}=\varepsilon_{\mathrm{I}}$ & 0.025 & 0.025 & 0.05 \\
\hline Computing time $(s)$ & 31 & 50 & 13 \\
\hline Volume of $\widehat{\mathscr{X}}(10)$ & 0.0034 & 0.0021 & 0.0035
\end{tabular}

Table 1. Simulation results for recursive state estimation

sets coincide with the lower bounds of the corrected sets). All computations have been performed with $\varepsilon_{\mathrm{S}}=\varepsilon_{\mathrm{I}}$ on an Athlon $1800+$ and the results are summarized in Table 1 .

In both cases, $90 \%$ of the computing time is spent during the first prediction step, when the knowledge about the initial value of the state is poor; the last steps take less than $0.1 \mathrm{~s}$ each. The enclosures obtained in Case $b$ are more accurate than in Case $a$ for the same value of $\varepsilon_{\mathrm{S}}$ and $\varepsilon_{\mathrm{I}}$, and obtained much faster when a given final precision is required. These results illustrate the efficiency of the bounding approach using cooperative systems.
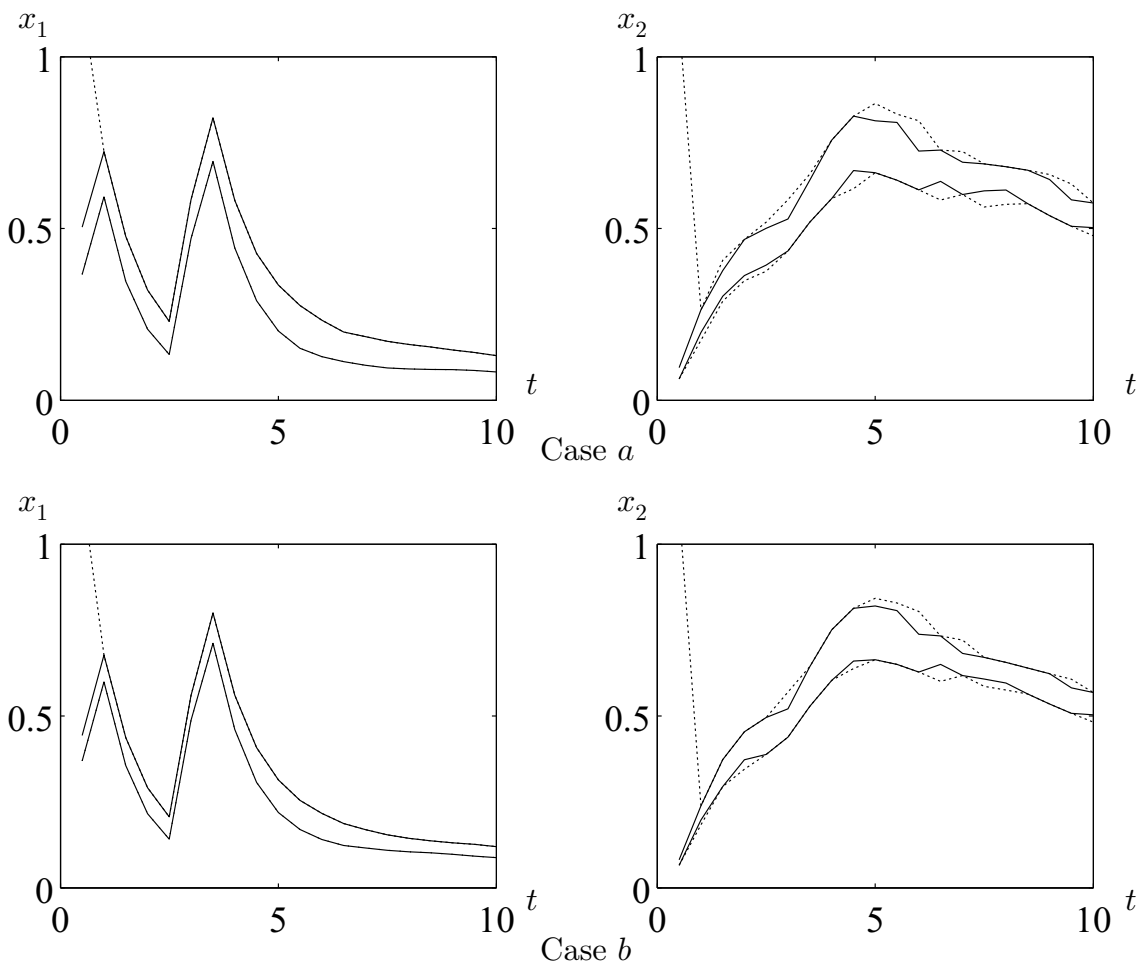

Fig. 5. Recursive bounded-error state estimation; lower and upper bounds of the smallest boxes enclosing the predicted set (dashed line) and corrected set (solid line); Case $a$ : direct guaranteed integration of the model; Case $b$ : guaranteed integration of the bounding cooperative systems 


\section{Bounded-error parameter estimation}

In this section, an estimate $\widehat{\mathbf{p}}$ for $\mathbf{p}$ is to be obtained such that the output $\mathbf{y}_{\mathrm{m}}(\mathbf{x}(t), \mathbf{p}, t)$ of the model (1) - (3) is an acceptable approximation of the output $\mathbf{y}(t)$ of the system. Here, the state vector $\mathbf{x}(t)$, if it is only known to belong to a given box $[\mathbf{x}(t)]$, plays the role of the nuisance uncertain quantity that is not estimated.

\subsection{Introduction}

Standard parameter estimation techniques (see, e.g., [28] and the references therein) compute $\widehat{\mathbf{p}}$ as the argument of the minimum of a given cost function, e.g.,

$$
j(\mathbf{p})=\left(\mathbf{y}-\mathbf{y}_{\mathrm{m}}(\mathbf{p})\right)^{\mathrm{T}}\left(\mathbf{y}-\mathbf{y}_{\mathrm{m}}(\mathbf{p})\right),
$$

where

$$
\mathbf{y}=\left(\mathbf{y}^{\mathrm{T}}\left(t_{1}\right), \ldots, \mathbf{y}^{\mathrm{T}}\left(t_{N}\right)\right)^{\mathrm{T}}
$$

and

$$
\mathbf{y}_{\mathrm{m}}(\mathbf{p})=\left(y_{\mathrm{m}}^{\mathrm{T}}\left(\mathbf{x}\left(t_{1}\right), \mathbf{p}, t_{1}\right), \ldots, y_{\mathrm{m}}^{\mathrm{T}}\left(\mathbf{x}\left(t_{N}\right), \mathbf{p}, t_{N}\right)\right)^{\mathrm{T}}
$$

are the system and model outputs collected at given time instants $t_{i}, i=1, \ldots, N$. This minimization can be performed by local-search algorithms such as Gauss-Newton or Levenberg-Marquardt, but there is no guarantee of convergence to a global minimizer of $j(\mathbf{p})$ and this minimizer may even not be unique. Random search, using, e.g., simulated annealing or genetic algorithms cannot provide any guarantee either that the global minimum has been found after finite computations. Only global guaranteed techniques, such as Hansen's algorithm [7], based on interval analysis, can obtain such guaranteed results.

Parameter bounding is an alternative approach searching for the set of all parameter vectors that are consistent with the experimental data, model structure and error bounds. It is similar to the correction step involved in the recursive state estimation algorithm presented in Section 2.2.

\subsection{Principle}

With the same hypotheses as in Section 2.2, the parameter vector $\mathbf{p} \in\left[\mathbf{p}_{0}\right]$ is deemed acceptable if the difference between the ouput $\mathbf{g}\left(\mathbf{x}\left(t_{i}\right), \mathbf{p}\right)$ of the deterministic part of the model and the experimental datum $\mathbf{y}\left(t_{i}\right)$ remains in $\left[\underline{\mathbf{v}}_{i}, \overline{\mathbf{v}}_{i}\right]$ for all $i=1, \ldots, N$. Parameter estimation then amounts to characterizing the set $\mathbb{P}$ of all acceptable $\mathbf{p} \in\left[\mathbf{p}_{0}\right]$

$$
\mathbb{P}=\left\{\mathbf{p} \in\left[\mathbf{p}_{0}\right] \mid \mathbf{y}\left(t_{i}\right)-\mathbf{g}\left(\mathbf{x}\left(t_{i}\right), \mathbf{p}\right) \in\left[\underline{\mathbf{v}}_{i}, \overline{\mathbf{v}}_{i}\right], i=1, \ldots, N\right\} .
$$

When the observation equation (3) reduces to

$$
\mathbf{y}_{\mathrm{m}}(\mathbf{p}, t)=\mathbf{h}(\mathbf{p}, t)+\mathbf{v}(t)
$$

with $\mathbf{h}(\mathbf{p}, t)$ some closed-form expression where $\mathbf{x}(t)$ does not appear, then the way $\mathbb{P}$ may be characterized depends mainly on whether $\mathbf{h}(\mathbf{p}, t)$ is linear in $\mathbf{p}$. If it is, $\mathbb{P}$ is a 
polytope that may be described exactly [27] or by an outer-approximation for instance using ellipsoids [3], [25]. When $\mathbf{h}(\mathbf{p}, t)$ is nonlinear in $\mathbf{p}, \mathbb{P}$ is no longer a polytope and may even be disconnected. One may nevertheless get a guaranteed enclosure of $\mathbb{P}$ using SIVIA.

When no closed-form solution of the model equations is available, again numerical integration has to be put at work to compute a box $\left[\mathbf{x}\left(t_{i}\right)\right]$ containing the state at each $t_{i}$ in order to enclose $\mathbf{g}\left(\mathbf{x}\left(t_{i}\right), \mathbf{p}\right)$. The box $\left[\mathbf{x}\left(t_{i}\right)\right]$ is obtained efficiently when (1) can be bounded between two cooperative systems as in Section 2.3.

The characterization of $\mathbb{P}$ is then realized using SIVIA, as presented in Section 2.3. The main difference is that bisection is now performed in $\mathbf{p}$-space instead of $\mathbf{x}$-space.

\subsection{Example}

Consider the same example as in Section 2.4, and suppose now that the initial state is perfectly known $\mathbf{x}_{0}=(0,0)^{\mathrm{T}}$ and that only the last two components of the parameter vector are known, the first two $\left(p_{1}, p_{2}\right)$ being only known to belong to $[0,5] \times[0,5]$.

Data have been obtained using the same simulation conditions as in Section 2.4. To evaluate an inclusion function for the state, the two bounding cooperative systems are now

$$
\left\{\begin{array}{l}
x_{1}^{\prime}=-\frac{\bar{p}_{1} x_{1}}{1+\underline{p}_{2} x_{1}}-p_{3}^{*} x_{1}+p_{4}^{*} x_{2}+u, \\
x_{2}^{\prime}=\frac{\underline{p}_{1} x_{1}}{1+\bar{p}_{2} x_{1}}-p_{4}^{*} x_{2},
\end{array}\right.
$$

and

$$
\left\{\begin{array}{l}
x_{1}^{\prime}=-\frac{\underline{p}_{1} x_{1}}{1+\bar{p}_{2} x_{1}}-p_{3}^{*} x_{1}+p_{4}^{*} x_{2}+u, \\
x_{2}^{\prime}=\frac{\bar{p}_{1} x_{1}}{1+\underline{p}_{2} x_{1}}-p_{4}^{*} x_{2},
\end{array}\right.
$$

The problem is now to evaluate the set of all parameter values $\left(p_{1}, p_{2}\right)$ that are compatible with the collected data and their associated error bounds (see Figure 4). The SIVIA algorithm has been used with initial search box $[0,5] \times[0,5]$ in parameter space. Guaranteed integration is again performed with the help of VNODE. With $\varepsilon_{\mathrm{S}}=0.05$, the subpaving represented on Figure 6 has been obtained in $400 \mathrm{~s}$ on an Athlon 1800+. It contains the actual values of the parameters $\left(p_{1}^{*}, p_{2}^{*}\right)=(1,4 / 3)$.

\section{Conclusions}

This paper presents an alternative and guaranteed approach for parameter and state estimation for continuous-time nonlinear differential models in a context of bounded errors with known bounds. An outer-approximation of the set of all parameter or state vectors that are consistent with the model structure and experimental data is obtained.

The only requirement is that the dynamical state equation of the system can be bounded between two cooperative systems. This is the case for all compartment models 


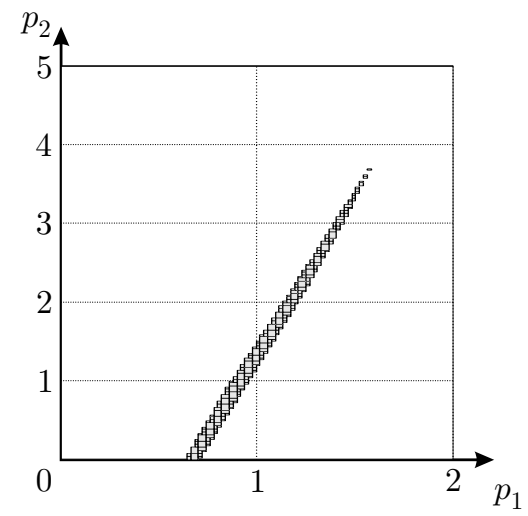

Fig. 6. Parameter estimation; solution subpaving in the $\left(p_{1}, p_{2}\right)$-plane when $\varepsilon=0.025$

and for many other positive systems, i.e., systems for which the state and parameters are constrained to remain positive.

The benefit of the enclosure between cooperative systems has been illustrated on an example. An ODE with uncertain parameters is replaced by two bounding ODEs with known parameters, the integration of which can be performed much more accurately, eliminating the wrapping effect.

\section{References}

1. V. Alcaraz-González, A. Genovesi, J. Harmand, A. González, A. Rapaport, and J. Steyer. Robust exponential nonlinear interval observer for a class of lumped models useful in chemical and biochemical engineering. Application to a wastewater treatment process. In Proc. MISC'99 Workshop on Applications of Interval Analysis to Systems and Control, pages 225235, Girona, February 24-26, 1999.

2. F. L. Chernousko. State Estimation for Dynamic Systems. CRC Press, Boca Raton, FL, 1994.

3. E. Fogel and Y. F. Huang. On the value of information in system identification - bounded noise case. Automatica, 18(2):229-238, 1982.

4. A. Gelb. Applied Optimal Estimation. MIT Press, Cambridge, MA, 1974.

5. K. Godfrey. Compartimental Models and Their Application. Academic Press, London, 1983.

6. J. L. Gouzé, A. Rapaport, and Z. M. Hadj-Sadok. Interval observers for uncertain biological systems. Journal of Ecological Modelling, (133):45-56, 2000.

7. E. R. Hansen. Global Optimization Using Interval Analysis. Marcel Dekker, New York, NY, 1992.

8. J. Hoefkens, M. Berz, and K. Makino. Efficient high-order methods for ODEs and DAEs. In G. Corliss, C. Faure, and A. Griewank, editors, Automatic Differentiation : From Simulation to Optimization, pages 341-351, New-York, NY, 2001. Springer-Verlag.

9. J. Hoefkens, M. Berz, and K. Makino. Verified high-order integration of DAEs and ODEs. In W. Kraemer and J. W. von Gudenberg, editors, Scientific Computing, Validated Numerics, Interval Methods, pages 281-292, Boston, 2001. Kluwer.

10. L. Jaulin. Nonlinear bounded-error state estimation of continuous-time systems. Automatica, 38:1079-1082, 2002. 
11. L. Jaulin, M. Kieffer, I. Braems, and E. Walter. Guaranteed nonlinear estimation using constraint propagation on sets. International Journal of Control, 74(18):1772-1782, 2001.

12. L. Jaulin, M. Kieffer, O. Didrit, and E. Walter. Applied Interval Analysis. Springer-Verlag, London, 2001.

13. L. Jaulin and E. Walter. Guaranteed nonlinear parameter estimation from bounded-error data via interval analysis. Mathematics and Computers in Simulation, 35(2):123-137, 1993.

14. L. Jaulin and E. Walter. Set inversion via interval analysis for nonlinear bounded-error estimation. Automatica, 29(4):1053-1064, 1993.

15. R. E. Kalman. A new approach to linear filtering and prediction problems. Transactions of the AMSE, Part D, Journal of Basic Engineering, 82:35-45, 1960.

16. M. Kieffer. Estimation ensembliste par analyse par intervalles, application à la localisation d'un véhicule. PhD thesis, Université Paris-Sud, Orsay, France, 1999.

17. M. Kieffer, L. Jaulin, I. Braems, and E. Walter. Guaranteed set computation with subpavings. In W. Kraemer and J. W. von Gudenberg, editors, Scientific Computing, Validated Numerics, Interval Methods, pages 167-178, Boston, 2001.

18. M. Kieffer, L. Jaulin, and E. Walter. Guaranteed recursive nonlinear state bounding using interval analysis. International Journal of Adaptative Control and Signal Processing, 6(3):193-218, 2002.

19. M. Kieffer and E. Walter. Guaranteed nonlinear state estimator for cooperative systems. In Proceedings of SCAN 2002, Paris, 2002. (submitted to).

20. A. Kurzhanski and I. Valyi. Ellipsoidal Calculus for Estimation and Control. Birkhäuser, Boston, MA, 1997.

21. R. Lohner. Enclosing the solutions of ordinary initial and boundary value problems. In E. Kaucher, U. Kulisch, and C. Ullrich, editors, Computer Arithmetic: Scientific Computation and Programming Languages, pages 255-286. BG Teubner, Stuttgart, 1987.

22. R. Lohner. Computation of guaranteed enclosures for the solutions of ordinary initial and boundary value-problem. In J. R. Cash and I. Gladwell, editors, Computational Ordinary Differential Equations, pages 425-435, Oxford, 1992. Clarendon Press.

23. M. Milanese, J. Norton, H. Piet-Lahanier, and E. Walter, editors. Bounding Approaches to System Identification. Plenum Press, New York, NY, 1996.

24. N. S. Nedialkov and K. R. Jackson. Methods for initial value problems for ordinary differential equations. In U. Kulisch, R. Lohner, and A. Facius, editors, Perspectives on Enclosure Methods, pages 219-264, Vienna, 2001. Springer-Verlag.

25. F. C. Schweppe. Uncertain Dynamic Systems. Prentice-Hall, Englewood Cliffs, NJ, 1973.

26. H. L. Smith. Monotone Dynamical Systems: An Introduction to the Theory of Competitive and Cooperative Systems, volume 41 of Mathematical Surveys and Monographs. American Mathematical Society, Providence, RI, 1995.

27. E. Walter and H. Piet-Lahanier. Exact recursive polyhedral description of the feasible parameter set for bounded-error models. IEEE Transactions on Automatic Control, 34(8):911-915, 1989.

28. E. Walter and L. Pronzato. Identification of Parametric Models from Experimental Data. Springer-Verlag, London, 1997. 